\title{
FOLFIRINOX Versus Gemcitabine-based Therapy for Pancreatic Ductal Adenocarcinoma: Lessons from Patient-derived Cell Lines
}

\author{
SEBASTIAN K.S. BEGG ${ }^{1 *}$, DAVID J. BIRNBAUM ${ }^{1 * \#}$, JEFFREY W. CLARK ${ }^{2}$, \\ MARI MINO-KENUDSON ${ }^{3}$, ULRICH F. WELLNER ${ }^{4}$, OLIVER SCHILLING $^{5}$, KEITH D. LILLEMOE $^{1}$, \\ ANDREW L. WARSHAW ${ }^{1}$, CARLOS FERNÁNDEZ-DEL CASTILLO ${ }^{1}$ and ANDREW S. LISS ${ }^{1}$ \\ Departments of ${ }^{1}$ Surgery, ${ }^{2}$ Hematology/Oncology, and ${ }^{3}$ Pathology, \\ Massachusetts General Hospital and Harvard Medical School, Boston, MA, U.S.A.; \\ ${ }^{4}$ Department of Surgery, University Medical Center Schleswig-Holstein, Campus Lübeck, Lübeck, Germany; \\ ${ }^{5}$ Institute of Surgical Pathology, University Medical Center Freiburg, \\ Faculty of Medicine, University of Freiburg, Freiburg, Germany
}

\begin{abstract}
Background/Aim: FOLFIRINOX [fluorouracil (5$F U)$, irinotecan, oxaliplatin] and gemcitabine plus nabpaclitaxel are standard treatments for patients with pancreatic ductal adenocarcinoma (PDAC). Despite efficacy rates of less than $32 \%$, evidence is lacking to guide the use of one drug over the other. Herein, we compared the sensitivity of patient-derived PDAC cell lines to each of these regimens. Materials and Methods: Changes in the growth of 19 low-passage patientderived PDAC cell lines were evaluated in response to treatment with FOLFIRINOX and gemcitabine plus paclitaxel (Gem-Pac). Results: Six cell lines exhibited optimal sensitivity (high $E_{M a x}$ and low $\mathrm{GI}_{50}$ ) to FOLFIRINOX and three cell lines exhibited optimal sensitivity to Gem-Pac. Several cell lines that were optimally sensitive to one drug regimen exhibited very poor response to the other. Conclusion: Further characterization of cancer cells exhibiting preferential sensitivity to each of these regimens may allow the identification of biomarkers to guide the selection of appropriate chemotherapy for a given patient.
\end{abstract}

This article is freely accessible online.

*These Authors contributed equally to this study.

${ }^{\#}$ Current Affiliation: Department of Digestive Surgery, AixMarseille University, Marseille, France.

Correspondence to: Andrew S. Liss, Department of Surgery, Massachusetts General Hospital, 55 Fruit Street, Boston, MA, 02114, U.S.A. Tel: +1 6177266194, Fax: +1 6177242924, email: aliss@mgh.harvard.edu

Key Words: PDAC, pancreatic cancer, chemosensitivity, FOLFIRINOX, gemcitabine, paclitaxel.
Over the last decade, significant progress has been made in the treatment of pancreatic ductal adenocarcinoma (PDAC). While complete surgical resection remains the only curative treatment, advances in chemotherapy have helped improve patient outcomes and median overall survival. Currently, the two most commonly employed regimens are FOLFIRINOX and gemcitabine plus nanoparticle albumin-bound (nab) paclitaxel (Abraxane); $31.6 \%$ and $23 \%$ of patients who undergo neoadjuvant treatment with these agents, respectively, demonstrate a response to therapy $(1,2)$.

FOLFIRINOX is a combination therapy consisting of fluorouracil (5-FU), irinotecan, oxaliplatin, and a drug that enhances the efficacy of 5-FU, leucovorin. FOLFIRINOX targets DNA replication, at least in part, by cross-linking DNA (oxaliplatin) and inhibiting the activities of thymidylate synthase (5-FU) and toposimerase 1 (irinotecan) (3). In contrast, gemcitabine and paclitaxel target unique pathways: gemcitabine is a self-potentiating nucleoside analog that functions to inhibit DNA synthesis, and paclitaxel stabilizes microtubules leading to a defective mitotic process and cell death $(4,5)$. While paclitaxel was initially modified with albumin to eliminate the need for an allergy-inducing solvent required for taxol solubility, albumin may also enhance delivery to stroma-rich tumors and increase gemcitabine concentrations inside cancer cells (6).

Both combination therapies have become standard treatments in patients with advanced pancreatic cancer. In the neoadjuvant setting, they are associated with improved tumor down-staging, converting initially unresectable tumors into successful resections with R0 margins $(1,2,7)$. However, no clear evidence exists to support the use of one drug regimen over the other in any given patient (8-12). Since less than a third of patients exhibit a response to these treatments, the 
ability to identify a preferential response to one drug regimen versus another for a given patient would be highly desirable. The aim of this study was to evaluate and stratify the sensitivity of low-passage PDAC cell lines derived from primary and metastatic tumors to these regimens and investigate the role of the tumor microenvironment in cell line sensitivity.

\section{Materials and Methods}

Chemotherapeutic preparation. Irinotecan, 5-FU, oxaliplatin, leucovorin, gemcitabine, and paclitaxel were obtained from Selleckchem (Houston, TX, USA). The drugs were dissolved in dimethyl sulfoxide (DMSO) or nuclease-free water and stored at $-80^{\circ} \mathrm{C}$. Drugs constituting FOLFIRINOX and gemcitabine plus paclitaxel (Gem-Pac) were combined in the following molar ratios analogous to those used in patients; FOLFIRINOX: 1.00 irinotecan, 80.95 5-FU, 0.80 oxaliplatin, 1.07 leucovorin; Gem-Pac: 1.00 gemcitabine, 0.04 paclitaxel $(2,13)$.

Patient samples and clinical data. Cell lines were established from xenograft tumors derived from surgically resected primary and metastatic PDAC tumors. The chemosensitivities of 19 patientderived low-passage PDAC cell lines were tested. Patient clinical data corresponding to each PDAC cell line was derived from a prospective clinical database. Patients gave written, informed consent and the study was approved by our institutional review committee.

Cell culture. Cells were maintained in a humidified incubator at $37^{\circ} \mathrm{C}$ with $5 \% \mathrm{CO}_{2}$ and grown in DMEM/F-12 media containing $10 \%$ fetal bovine serum and $1 \%$ antibiotic-antimycotic. Conditioned media was prepared by plating cells at $3.0 \times 10^{6}$ cells per 10 centimeter cell culture dish. Media was changed $24 \mathrm{~h}$ after plating and conditioned media were collected after an additional $24 \mathrm{~h}$ of incubation. Conditioned media was cleared by centrifugation at $1,000 \mathrm{RPM}$ for five minutes at $4^{\circ} \mathrm{C}$ prior to storage at $-80^{\circ} \mathrm{C}$.

Cellular growth evaluation. Cell lines were plated in triplicate on 96well plates at a density of 2,000 cells per well. After $24 \mathrm{~h}$ of incubation, serial dilutions of FOLFIRINOX (ranging from $6 \times 10^{-5} \mu \mathrm{Mol}$ to 0.4 $\mu \mathrm{Mol}$ for irinotecan, $5 \times 10^{-3} \mu \mathrm{Mol}$ to $32.3 \mu \mathrm{Mol}$ for $5-\mathrm{FU}, 5 \times 10^{-5}$ $\mu \mathrm{Mol}$ to $0.32 \mu \mathrm{Mol}$ for oxaliplatin, and $6 \times 10^{-5} \mu \mathrm{Mol}$ to $0.42 \mu \mathrm{Mol}$ for leucovorin), Gem-Pac (ranging from $5 \times 10^{-6} \mu \mathrm{Mol}$ to $0.31 \mu \mathrm{Mol}$ for gemcitabine and $2 \times 10^{-7} \mu \mathrm{Mol}$ to $0.014 \mu \mathrm{Mol}$ for paclitaxel), gemcitabine alone (ranging from $4 \times 10^{-3} \mu \mathrm{Mol}$ to $1.25 \mu \mathrm{Mol}$ ), and paclitaxel alone (ranging from $2 \times 10^{-7} \mu \mathrm{Mol}$ to $0.014 \mu \mathrm{Mol}$ ) were added to the cells. These concentrations were selected based on the prior irinotecan reference concentration used to treat human colon cancer cell lines, LoVo (14). In a clinical setting, the 5-FU component of FOLFIRINOX is administered in an initial bolus followed by a continuous IV infusion over $46 \mathrm{~h}$, whereas, here, the chemotherapeutics were consolidated into a single administration. After five days of incubation, cell viability was assessed with resazurin-based assays using a SpectraMax M5 plate reader. At least three independent experiments were conducted for each cell line.

Data analysis. Statistical analysis was performed using the GraphPad Prism 7.0 software (GraphPad Software Inc., La Jolla, $\mathrm{CA}, \mathrm{USA})$. The maximum growth inhibition $\left(\mathrm{E}_{\mathrm{Max}}\right)$ achieved at the highest concentration of drug tested and the concentration of drug needed to achieve $50 \%$ cellular growth inhibition $\left(\mathrm{GI}_{50}\right)$ of each cell line in response to chemotherapeutic treatment was determined from the dose-response data of at least three separate experiments performed in triplicate. Statistical significance was determined using Student's t-test, log-rank test, and Pearson's correlation using GraphPad Prism 7.0 software. Significance was defined as $p<0.05$.

Evaluation of drug interactions. The interaction between gemcitabine and paclitaxel was evaluated by isobolographic analysis, a dose-oriented geometric method of assessing drug interactions (15). The combination index (CI) values were calculated based on the following equation:

$C I=\left[\left(G I_{50}\right.\right.$ of Gem-Pac relative to Gemcitabine $) /\left(G_{50}\right.$ of Gemcitabine Alone $)]+\left[\left(G_{50}\right.\right.$ of Gem-Pac relative to Paclitaxel $) /\left(G I_{50}\right.$ of Paclitaxel Alone)]

By this calculation, $\mathrm{CI}>1.3$ indicates antagonism, $\mathrm{CI}=1.1-1.3$ indicates moderate antagonism, $\mathrm{CI}=0.9$ to 1.1 indicates an additive effect, $\mathrm{CI}=0.8-0.9$ indicates slight synergism, $\mathrm{CI}=0.6-0.8$ indicates moderate synergism, $\mathrm{CI}=0.4-0.6$ indicates synergism, and $\mathrm{CI}=0.2$ 0.4 indicates strong synergism. The interaction between 5 -FU, irinotecan, oxaliplatin, and leucovorin was not evaluated, since monotherapy with each of these agents is not of clinical interest.

\section{Results}

Evaluation of PDAC cell line chemosensitivity. We exposed 19 low-passage patient-derived PDAC cell lines (Table I) to increasing doses of FOLFIRINOX and gemcitabine plus paclitaxel (Gem-Pac) to investigate the inherent sensitivities of the cell lines. Representative dose-response curves for six cell lines are shown in Figure 1A. The maximum growth inhibition $\left(\mathrm{E}_{\mathrm{Max}}\right)$ achieved at the highest concentration of drug tested was calculated and the cell lines were defined as having high $(>75 \%)$, moderate $(50-75 \%)$, or low $(<50 \%) \mathrm{E}_{\mathrm{Max}}$. An $\mathrm{E}_{\mathrm{Max}}$ of $>50 \%$ was observed in each cell line in response to at least one of the two drug combinations (Figure 1B). All PDAC cell lines exhibited a high $(n=15,78.9 \%)$ or moderate $(\mathrm{n}=4,21.1 \%) \mathrm{E}_{\mathrm{Max}}$ in response to FOLFIRINOX. In contrast, cell lines exhibited a broad distribution of $\mathrm{E}_{\mathrm{Max}}$ in response to Gem-Pac; six cell lines (31.6\%) exhibited high $\mathrm{E}_{\mathrm{Max}}$, seven $(36.8 \%)$ exhibited moderate $\mathrm{E}_{\mathrm{Max}}$, and six (31.6\%) exhibited low $\mathrm{E}_{\mathrm{Max}}$. Eleven cell lines $(57.9 \%)$ exhibited preferential maximum growth inhibition to FOLFIRINOX over Gem-Pac, while only one cell line (MGH1415) demonstrated a preferential response to Gem-Pac over FOLFIRINOX.

In addition to $\mathrm{E}_{\mathrm{Max}}$, the concentration of drug needed to achieve $50 \%$ cellular growth inhibition $\left(\mathrm{GI}_{50}\right)$ of each cell line in response to FOLFIRINOX and Gem-Pac was calculated relative to the concentrations of irinotecan and gemcitabine in each combination chemotherapy, respectively. Based on the $\mathrm{GI}_{50}$ values, cell lines were defined as having high ( $<11 \mathrm{nMol}$ FOLFIRINOX, <20 nMol Gem-Pac), moderate (14-400 nMol FOLFIRINOX, 30-314 nMol Gem- 
Table I. Histopathological features of PDAC cell lines.

\begin{tabular}{|c|c|c|c|c|c|c|}
\hline \multirow[b]{2}{*}{ Cell line } & \multirow[b]{2}{*}{ Gender } & \multirow[b]{2}{*}{ Age } & \multirow[b]{2}{*}{ Primary/Met } & \multirow[b]{2}{*}{ Grade } & \multicolumn{2}{|c|}{ Therapy } \\
\hline & & & & & Neoadjuvant & Adjuvant \\
\hline MGH617 & Male & 57 & Primary & III & None & None \\
\hline MGH1108 & Female & 73 & Liver Met & N/A & None & None \\
\hline MGH1152 & Female & 56 & Primary & II & None & None \\
\hline MGH1157 & Female & 62 & Primary & II & None & None \\
\hline MGH1222 & Female & 78 & Primary & II & None & None \\
\hline MGH1275 & Male & 70 & Liver Met & N/A & None & None \\
\hline MGH1289 & Male & 56 & Primary & I & None & Gem + Cap \\
\hline MGH1294 & Female & 37 & Primary & II & None & None \\
\hline MGH1300 & Female & 87 & Primary & N/A & None & None \\
\hline MGH1309 & Male & 62 & Primary & III & None & None \\
\hline MGH1312 & Male & 51 & Liver Met & III & None & None \\
\hline MGH1319 & Male & 66 & Primary & III & None & None \\
\hline MGH1326 & Female & 82 & Primary & II & None & None \\
\hline MGH1415 & Male & 64 & Liver Met & N/A & None & None \\
\hline MGH1473 & Male & 61 & Lung Met & N/A & None & None \\
\hline MGH1498 & Female & 68 & Primary & III & None & None \\
\hline MGH1513 & Male & 64 & Primary & II & Cap + R.T. & None \\
\hline MGH1925 & Female & 75 & Peritoneal Met & II & None & FX + LDE225 \\
\hline MGH1968 & Male & 79 & Primary & II & None & None \\
\hline
\end{tabular}

Gem: Gemcitabine; Cap: capecitabine; R.T.: radiation therapy; FX: FOLFIRINOX.

Pac), or low (>400 nMol FOLFIRINOX, >314 nMol Gem$\mathrm{Pac}$ ) sensitivity to these chemotherapeutics (Figure 1C). In general, the growth of PDAC cell lines was more broadly sensitive to FOLFIRINOX than to Gem-Pac. Only one cell line $(5.3 \%)$ exhibited low sensitivity to FOLFIRINOX compared to the six cell lines (31.6\%) with low sensitivity to Gem-Pac. The cell line (MGH1498) that exhibited low sensitivity to FOLFIRINOX also exhibited low sensitivity to Gem-Pac. Two cell lines (MGH1289 and MGH1294) exhibited both high sensitivity to FOLFIRINOX and low sensitivity to Gem-Pac.

While $\mathrm{E}_{\mathrm{Max}}$ and $\mathrm{GI}_{50}$ individually provided valuable insight into the response of cells to FOLFIRINOX and Gem-Pac, the evaluation of both metrics in combination allowed us to identify cell lines that we classified as optimally sensitive to these chemotherapeutic combinations. Optimal sensitivity was defined as a combination of high $\mathrm{E}_{\mathrm{Max}}(>75 \%)$ and low $\mathrm{GI}_{50}$ ( $<11 \mathrm{nMol}$ FOLFIRINOX, $<20 \mathrm{nMol}$ Gem-Pac). Six cell lines (MGH1108, MGH1222, MGH1289, MGH1294, MGH1300, and MGH1312) exhibited optimal sensitivity to FOLFIRINOX (31.6\%) while three cell lines (MGH1108, MGH1312, and MGH1415) exhibited optimal sensitivity to Gem-Pac (15.8\%) (Figure 2). Two cell lines (MGH1108 and MGH1312) had similar sensitivities to these two regimens.

Characterization of gemcitabine and paclitaxel interaction. Gemcitabine had historically been used as a first-line therapy in the treatment of PDAC (2). While gemcitabine plus nanoparticle albumin-bound paclitaxel has replaced gemcitabine alone as a standard neoadjuvant chemotherapy option for patients with PDAC, limited research has been conducted regarding the efficacy of paclitaxel monotherapy $(6,16)$. To better understand the role of gemcitabine and paclitaxel in combination, the effect of the individual drugs was evaluated in six cell lines that exhibited high $\mathrm{GI}_{50}$ in response to Gem-Pac. Given the different dilution ranges used to treat cells with Gem-Pac and gemcitabine alone, a reference dose of $0.31 \mu \mathrm{Mol}$ of gemcitabine was used to calculate $\mathrm{E}_{\mathrm{Max}}$ for cell line response to both drugs. Dose-response curves generated for each cell line indicated that paclitaxel was the primary mediator of Gem-Pac sensitivity in terms of both $\mathrm{E}_{\mathrm{Max}}$ and $\mathrm{GI}_{50}$ (Figure $3 \mathrm{~A}$ and $\mathrm{B}$ ). In regard to $\mathrm{E}_{\mathrm{Max}}$, paclitaxel drove the inhibitory effects of Gem-Pac in two cell lines (MGH1108 and MGH1473), gemcitabine drove the inhibitory effects of Gem-Pac in one cell line (MGH1415), and the remaining three cell lines (MGH1275, MGH1309, and MGH1312) exhibited similar $\mathrm{E}_{\mathrm{Max}}$ in response to Gem-Pac, gemcitabine alone, and paclitaxel alone (Figure 3C). In regard to $\mathrm{GI}_{50}$, all six cell lines exhibited a dramatically higher sensitivity to paclitaxel relative to Gem-Pac than to gemcitabine relative to Gem-Pac (Figure 3D). To better define the relationship between gemcitabine and paclitaxel in combination, the interaction between these drugs was evaluated by isobolographic analysis (15). The combination index (CI) values for the six treated cell lines indicated a synergistic interaction between gemcitabine and paclitaxel in 
A
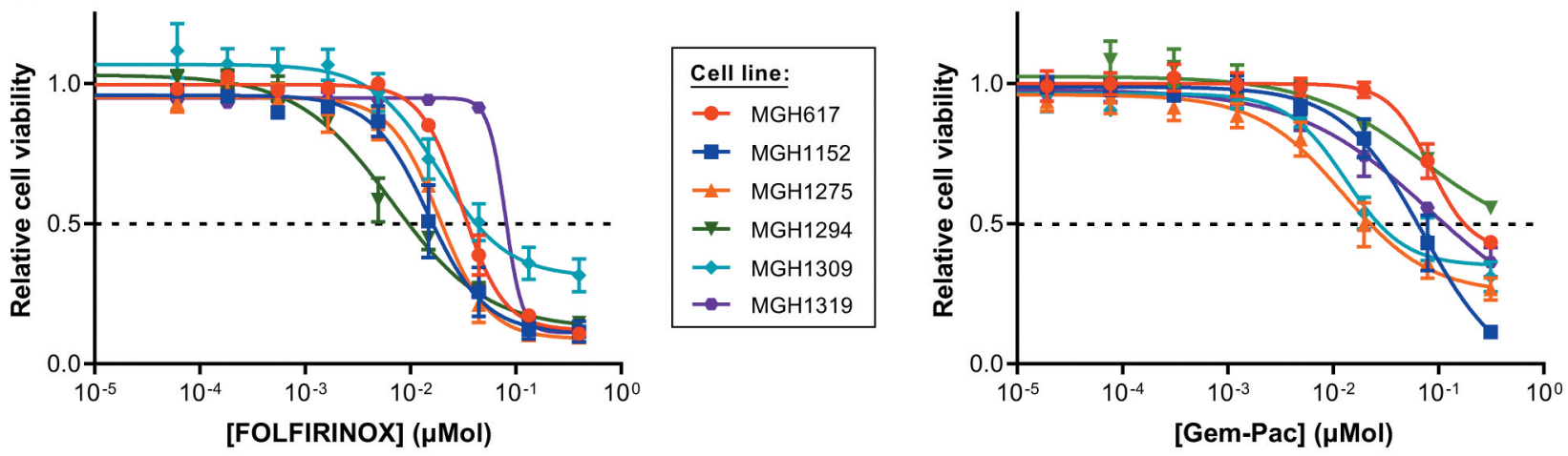

B

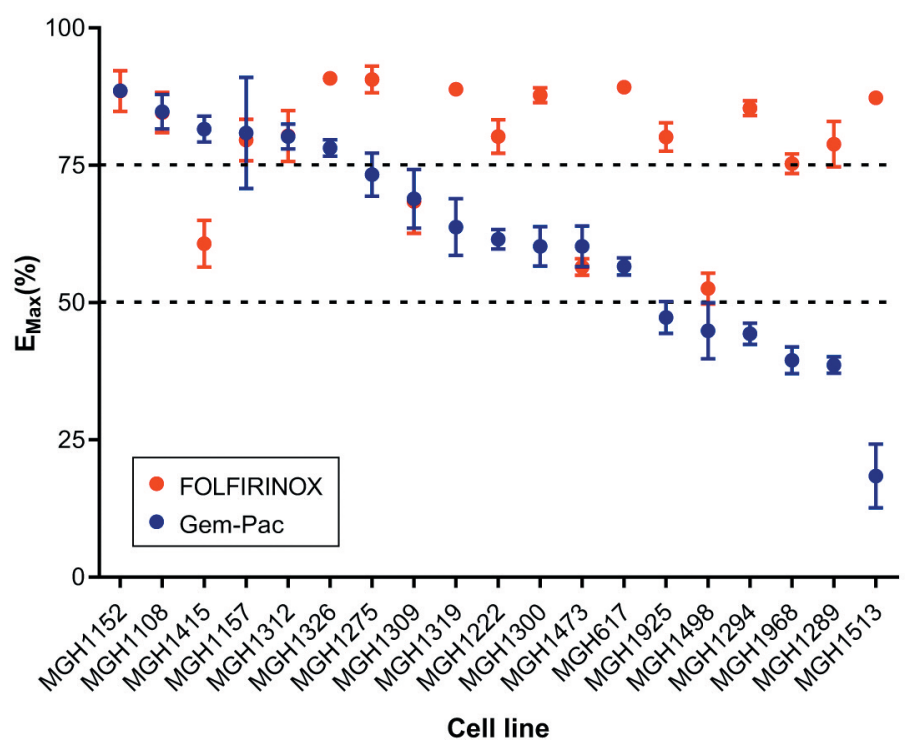

C

FOLFIRINOX

\begin{tabular}{|c|c|}
\hline Cell line & GI $_{\mathbf{5 0}}$ (nMol) \\
\hline MGH1108 & 2.48 \\
MGH1222 & 5.02 \\
MGH1294 & 6.67 \\
MGH1312 & 7.57 \\
MGH1300 & 10.25 \\
MGH1289 & 10.90 \\
\hline MGH1415 & 14.10 \\
MGH1309 & 14.71 \\
MGH1157 & 15.48 \\
MGH1513 & 16.54 \\
MGH1275 & 18.67 \\
MGH1152 & 18.88 \\
MGH1968 & 21.73 \\
MGH1326 & 29.86 \\
MGH617 & 30.91 \\
MGH1473 & 61.32 \\
MGH1319 & 79.55 \\
MGH1925 & 83.31 \\
\hline MGH1498 & $>400$ \\
\hline
\end{tabular}

Gem-Pac

\begin{tabular}{|c|c|}
\hline Cell line & $\mathbf{G l}_{\mathbf{5 0}}$ (nMol) \\
\hline MGH1108 & 4.64 \\
MGH1312 & 10.15 \\
MGH1275 & 12.50 \\
MGH1309 & 13.14 \\
MGH1473 & 17.82 \\
MGH1415 & 19.30 \\
\hline MGH1157 & 30.01 \\
MGH1300 & 41.49 \\
MGH1326 & 51.25 \\
MGH1222 & 57.98 \\
MGH1152 & 70.43 \\
MGH1319 & 80.68 \\
MGH617 & 83.87 \\
\hline MGH1289 & $>314$ \\
MGH1294 & $>314$ \\
MGH1498 & $>314$ \\
MGH1513 & $>314$ \\
MGH1925 & $>314$ \\
MGH1968 & $>314$ \\
\hline
\end{tabular}

Figure 1. The response of PDAC cell lines to chemotherapeutic treatment. (A) Representative dose-response curves for six PDAC cell lines (MGH617, MGH1152, MGH1275, MGH1294, MGH1309, MGH1319) treated with FOLFIRINOX (left) and Gem-Pac (right). Cellular growth was evaluated by resazurin assay after incubation for five days with treatment. $(B)$ The maximum growth inhibition $\left(E_{M a x}\right)$ achieved at the highest concentration of drug tested for all 19 PDAC cell lines in response to treatment with FOLFIRINOX (red) and Gem-Pac (blue). (C) The concentration of drug needed to achieve 50\% cellular growth inhibition $\left(G_{50}\right)$ for all 19 PDAC cell lines in response to treatment with FOLFIRINOX (left) and GemPac (right). All results are presented as the average \pm standard error of the mean from at least three experiments performed in triplicate.

three cell lines (MGH1108, MGH1275, and MGH1473; $\mathrm{CI}=0.32-0.52)$ and an additive interaction between gemcitabine and paclitaxel in the remaining three cell lines (MGH1309, MGH1312, MGH1415; CI=0.99-1.09).

The effect of the tumor microenvironment on chemosensitivity. PDAC is characterized by a dense stromal component in which cancer-associated fibroblasts (CAFs) comprise the majority of the non-neoplastic cells of the tumor (17). CAFs have been implicated as a key barrier to chemotherapeutic treatment of PDAC, in part by enhancing the chemoresistance of PDAC cells (17). To assess whether the chemosensitivity of PDAC cell lines to FOLFIRINOX and Gem-Pac is affected by CAFs, we employed two CAF lines derived from PDAC (CAF-1 and CAF-2) and one immortalized hepatic stellate cell line (LX-2). The hepatic-derived cell line was used to interrogate whether liver-derived fibroblasts likely found in liver metastases impart a differential effect on the chemosensitivity of PDAC cells.

Cell lines that exhibited optimal sensitivity to both FOLFIRINOX and Gem-Pac (MGH1108) or were exclusively optimally sensitive to FOLFIRINOX (MGH1222) or Gem-Pac (MGH1415) were treated with chemotherapeutics in the 
FOLFIRINOX

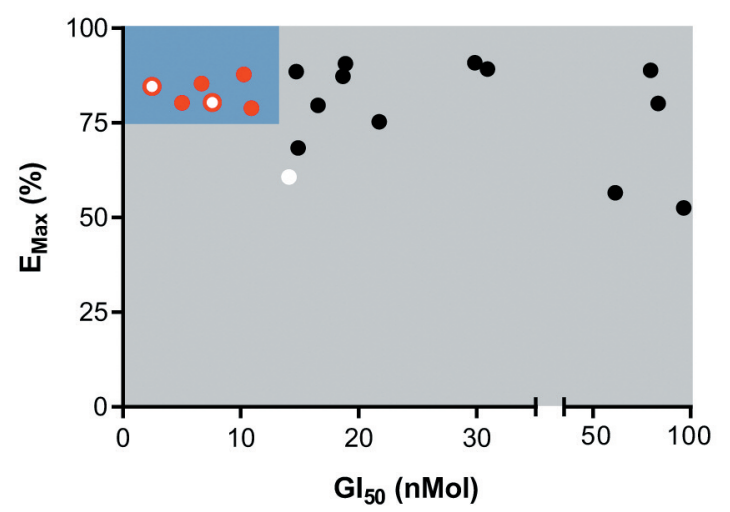

Gem-Pac

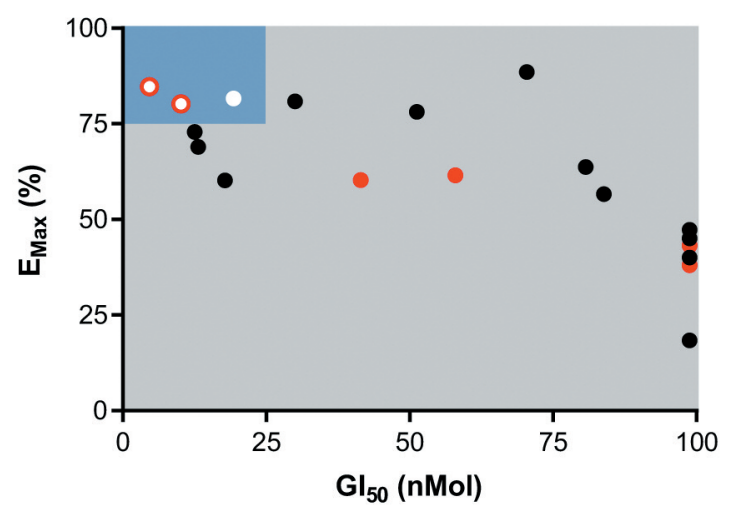

Optimal sensitivity to FOLFIRINOX alone

Optimal sensitivity to Gem-Pac alone
Optimal sensitivity to both drugs

Sub-optimal sensitivity to both drugs

Figure 2. Identification of PDAC cell lines exhibiting optimal sensitivity to chemotherapeutic treatment. The $E_{\text {Max }}$ and $G_{50}$ of each cell line were plotted. Cell lines exhibiting optimal sensitivity $\left(E_{\text {Max }}<75 \%\right.$ and $\left.G_{50}<11 \mathrm{nMol} \mathrm{FOLFIRINOX;<20} \mathrm{nMol} \mathrm{Gem-Pac}\right)$ are indicated by the blue box. Cell lines with unique optimal sensitivity to FOLFIRINOX are indicated by red circles (MGH1222, MGH1289, MGH1294, and MGH1300), and the cell line with unique optimal sensitivity to Gem-Pac is indicated by a white circle (MGH1415). Cell lines optimally sensitive to both FOLFIRINIOX and Gem-Pac are indicated by red and white circles (MGH1108 and MGH1312).

presence of conditioned media from each of the three fibroblast lines: CAF-1, CAF-2, and LX-2. While the $\mathrm{E}_{\mathrm{Max}}$ of cell lines treated with FOLFIRINOX was not altered by the presence of fibroblast-conditioned media, the sensitivity of MGH1108 cells $\left(\mathrm{GI}_{50}=2.38 \mathrm{nMol}\right)$ was dramatically decreased in the presence of conditioned media from one fibroblast line (CAF-2), with a ten-fold increase in $\mathrm{GI}_{50}$ observed $\left(\mathrm{GI}_{50}=22.26 \mathrm{nMol}\right.$; $p=0.014$ ) (Figure 4A). The sensitivity of MGH1222 cells to FOLFIRINOX was not affected by fibroblast conditioned media. The sensitivity of cell line MGH1108 to Gem-Pac $\left(\mathrm{GI}_{50}=4.28 \mathrm{nMol}\right)$ was decreased at least three-fold by conditioned media from CAF-1 $\left(\mathrm{GI}_{50}=13.44 \mathrm{nMol}\right), \mathrm{CAF}-2$ $\left(\mathrm{GI}_{50}=24.80 \mathrm{nMol}\right)$, and LX-2 $\left(\mathrm{GI}_{50}=16.31 \mathrm{nMol} ; p \leq 0.001\right)$ (Figure 4C). A similar three-fold decrease in sensitivity to Gem-Pac was also observed in cell line MGH1415 $\left(\mathrm{GI}_{50}=22.40 \mathrm{nMol}\right)$ when treated in LX-2 conditioned media $\left(\mathrm{GI}_{50}=70.07 ; p>0.001 \mathrm{nMol}\right.$ ) (Figure 4D). However, CAF-1 and CAF-2 conditioned media did not significantly alter the $\mathrm{GI}_{50}$ of MGH1415 cells in response to Gem-Pac.

FOLFIRINOX and Gem-Pac are non-selective chemotherapeutics that may also impact the tumor microenvironment. Given the importance of the stroma in affecting the chemosensitivity of PDAC cells, the ability of these drugs to reduce the viability of CAFs may have profound effects on the chemotherapeutic response of the tumor. To evaluate the sensitivity of fibroblasts to FOLFIRINOX and Gem-Pac and assess whether their chemosensitivity is affected by the presence of PDAC, fibroblast lines CAF-1, CAF-2, and LX-2 were treated with FOLFIRINOX and Gem-Pac in PDAC cell line conditioned media. Fibroblast lines exhibited a range of $\mathrm{E}_{\mathrm{Max}}(68.2 \%$ $85.6 \%$ ) and $\mathrm{GI}_{50}$ (FOLFIRINOX: $32.82 \mathrm{nMol}-95.44 \mathrm{nMol}$; Gem-Pac: $4.387 \mathrm{nMol}-10.98 \mathrm{nMol}$ ) in response to both drug combinations in unconditioned media that was comparable to the chemosensitivity of PDAC cells. When treated in PDAC cell line conditioned media, all fibroblast lines exhibited a decrease in sensitivity to both chemotherapeutics (Figure 5). A key difference in the ability of PDAC conditioned media to alter the chemotherapeutic response of fibroblasts was observed with $\mathrm{E}_{\mathrm{Max}}$; all three fibroblast lines exhibited a decrease in $\mathrm{E}_{\text {Max }}$ to FOLFIRINOX when treated with PDAC cell line conditioned media $(p<0.05)$, while the response of fibroblast lines to Gem-Pac was not affected under similar media conditions.

\section{Discussion}

FOLFIRINOX and gemcitabine plus Abraxane have become the two standard chemotherapeutic treatments in patients with metastatic PDAC. However, no evidence exists to guide the selection of one chemotherapy over the other for any given patient (8-12). This study found that patient-derived PDAC cell lines exhibit differential sensitivity to FOLFIRINOX and gemcitabine plus paclitaxel (Gem-Pac), reflecting the heterogeneity of patient response to these drug regimens; six of the 19 cell lines exhibited optimal sensitivity (high $\mathrm{E}_{\mathrm{Max}}$ 
A

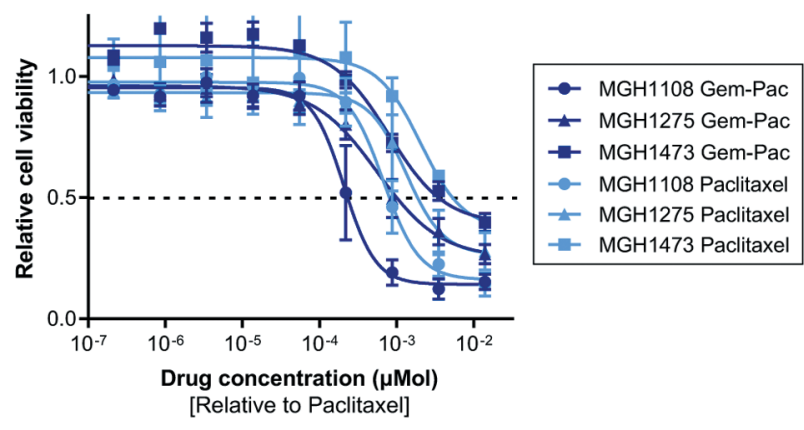

C

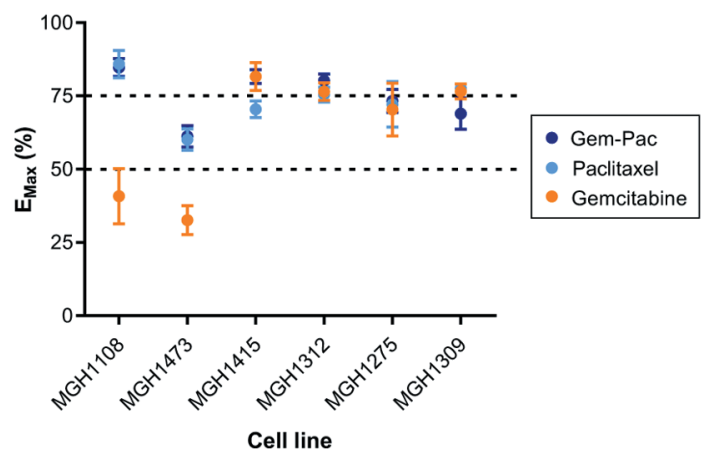

B

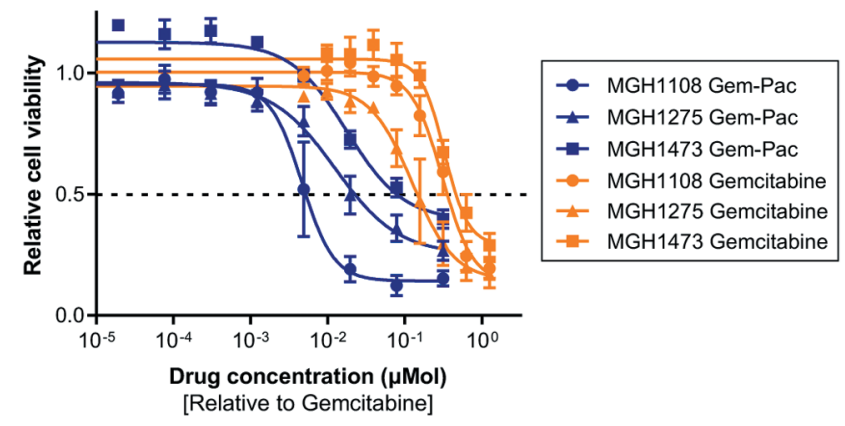

D

\begin{tabular}{|c|c|c|c|c|c|c|}
\hline \multirow[b]{2}{*}{ Cell line } & \multicolumn{2}{|c|}{$\begin{array}{l}\mathrm{Gl}_{50}(\mathrm{nMol}) \\
\text { Relative to Paclitaxel }\end{array}$} & \multicolumn{2}{|c|}{$\begin{array}{l}\qquad \mathrm{GI}_{50}(\mathrm{nMol}) \\
\text { Relative to Gemcitabine }\end{array}$} & \multirow[b]{2}{*}{$\mathrm{Cl}$} & \multirow[b]{2}{*}{ Relationship } \\
\hline & Paclitaxel & Gem-Pac & Gemcitabine & Gem-Pac & & \\
\hline MGH1108 & 0.67 & 0.21 & 307.40 & 4.64 & 0.32 & Strong synergism \\
\hline MGH1275 & 1.33 & 0.56 & 125.70 & 12.50 & 0.52 & Synergism \\
\hline MGH1309 & 0.69 & 0.59 & 65.12 & 13.14 & 1.06 & Additive effect \\
\hline MGH1312 & 0.52 & 0.45 & 44.61 & 10.15 & 1.09 & Additive effect \\
\hline MGH1415 & 1.42 & 0.86 & 50.24 & 19.30 & 0.99 & Additive effect \\
\hline MGH1473 & 2.07 & 0.80 & 325.90 & 17.82 & 0.44 & Synergism \\
\hline
\end{tabular}

Figure 3. The response of PDAC cell lines to gemcitabine and paclitaxel monotherapy. (A) Representative dose-response curves for three PDAC cell lines in response to treatment with Gem-Pac (dark blue) and paclitaxel alone (light blue). (B) Representative dose-response curves for three PDAC cell lines in response to treatment with Gem-Pac (dark blue) and gemcitabine alone (orange). (C) The $E_{M a x}$ of six PDAC cell lines in response to treatment with Gem-Pac (dark blue), paclitaxel alone (light blue), and gemcitabine alone (orange). A reference dose of 0.31 $\mu$ Mol of gemcitabine and $0.014 \mu \mathrm{Mol}$ of paclitaxel was used to calculate $E_{\text {Max }}$ for cell line response to Gem-Pac; a reference dose of $0.31 \mu M o l$ of gemcitabine was used to calculate $E_{\text {Max }}$ for cell line response to gemcitabine alone; and a reference dose of $0.014 \mu M o l$ of paclitaxel was used to calculate $E_{M a x}$ for cell line response to paclitaxel alone. (D) The $\mathrm{GI}_{50}$ of six PDAC cell lines in response to treatment with Gem-Pac, gemcitabine alone, and paclitaxel alone. Isobolographic analysis of these results yielded combination index (CI) values for the six treated cell lines.

and low $\mathrm{GI}_{50}$ ) in response to treatment with FOLFIRINOX, while three of 19 exhibited optimal sensitivity to Gem-Pac. Two cell lines had similar sensitivities to the two regimens, while the other cell lines were not responsive to either chemotherapy regimen. Several cell lines that were optimally sensitive to one drug regimen exhibited very poor response to the other, indicating that one chemotherapy was clearly superior. Additionally, the higher overall proportion of FOLFIRINOX-susceptible cell lines compared to Gem-Pacsusceptible cell lines is consistent with analysis of real-world clinical data suggesting that FOLFIRINOX may be overall more effective than gemcitabine and Abraxane chemotherapy for metastatic pancreatic cancer, although this has not yet been confirmed in a randomized study (18).

These findings warrant further investigation to reveal whether characteristics of each cell line are related to chemosensitivity or chemoresistance to one or the other chemotherapy regimen. It is unlikely that these effects are linked to simple genetic alterations; the four most commonly mutated genes in PDAC (KRAS, TP53, CDKN2A, and SMAD4) occur at higher rates than would account for these results, and additional genes are generally altered in less than $10 \%$ of tumors (19). However, a formal genetic analysis of these cell lines remains worthwhile. If the characteristics that define chemosensitivity or chemoresistance to one regimen or the other can be elucidated, the findings would have potential clinical implications. For example, knowing which chemotherapy is most effective for a given patient from the beginning of treatment could save precious time, potentially significantly improving the patient's clinical outcome. Likewise, knowing that two drug regimens are equally effective for a given patient could help direct the choice of a more tolerable chemotherapy and thereby improve the patient's quality of life. Finally, knowing which patients are unresponsive to chemotherapy altogether could spare the burden of a futile course of chemotherapy that would unnecessarily debilitate the patients' final stages of life. While these findings 
A

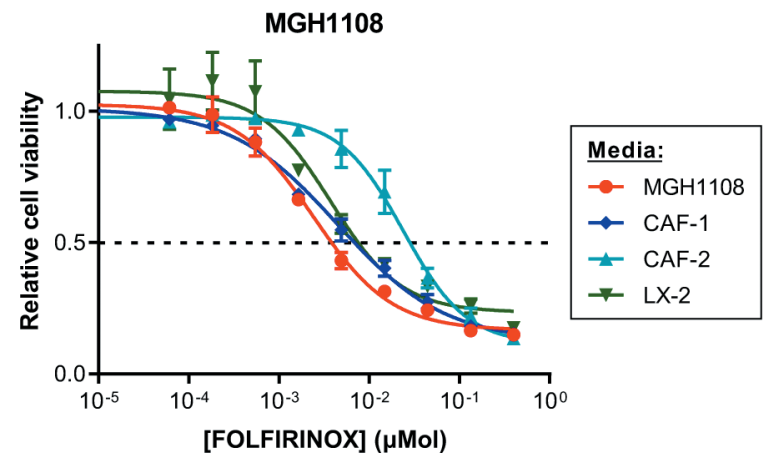

C

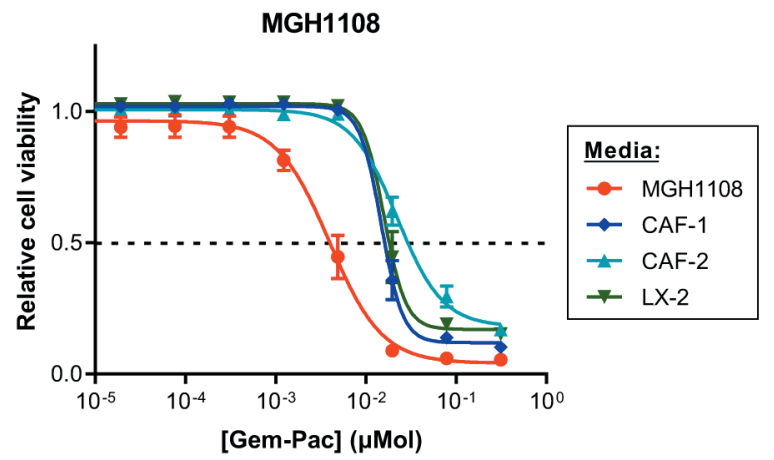

B

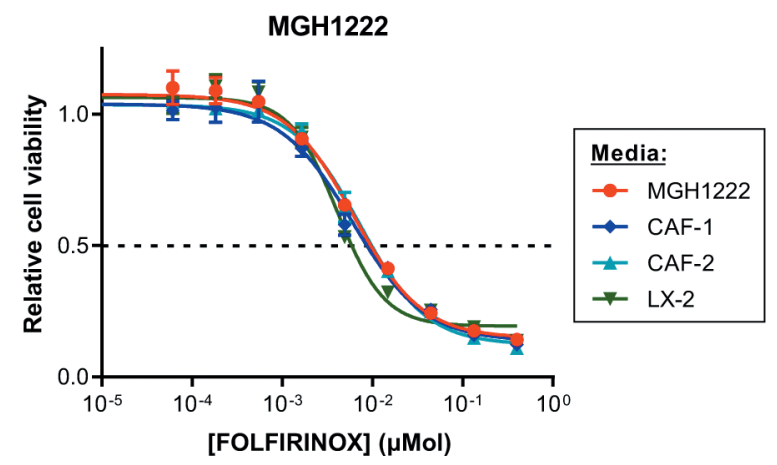

D

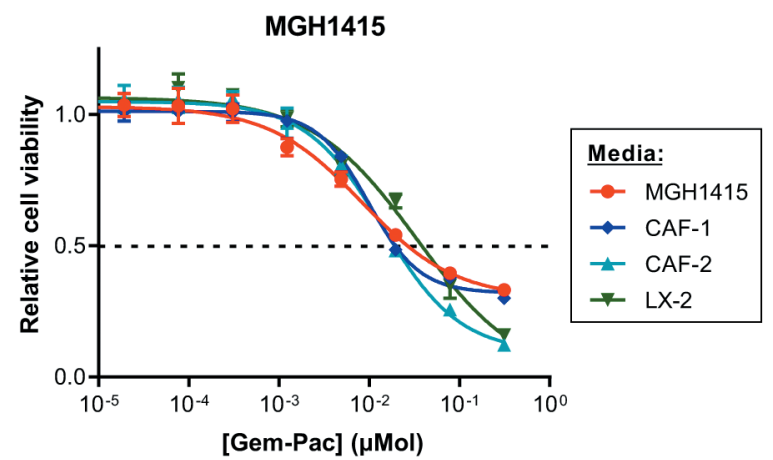

Figure 4. Effect of fibroblast-conditioned media on PDAC cell line chemosensitivity. (A) Dose-response curves for PDAC cell line MGH1108 treated with FOLFIRINOX in the presence of conditioned media from MGH1108 and conditioned media from two PDAC-derived CAF lines, CAF-1 and $C A F-2$, and the hepatic-derived fibroblast line LX-2. (B) Dose-response curves for PDAC cell line MGH1222 treated with FOLFIRINOX in the presence of conditioned media from MGH1222, CAF-1,CAF-2, and LX-2. (C) Dose-response curves for MGH1108 treated with gemcitabine plus paclitaxel (Gem-Pac) in the presence of conditioned media from MGH1108, CAF-1,CAF-2, and LX-2. (D) Dose-response curves for PDAC cell line MGH1415 treated with Gem-Pac in the presence of conditioned media from MGH1415, CAF-1, CAF-2, and LX-2.

constitute exciting preliminary results, it is based on a limited number of cell lines and additional studies are needed to extend this and define markers of chemosensitivity and resistance.

The examination of previously uninvestigated comparative effects of gemcitabine and paclitaxel monotherapy on patient derived cell lines revealed that paclitaxel drove the growth inhibitory effects in five of the six most Gem-Pac-susceptible cell lines. Interestingly, one of these cell lines was dramatically more sensitive to Gem-Pac than to gemcitabine and paclitaxel alone, suggesting that this combination exerts a synergistic effect on a subset of PDAC cells. These findings begin to elucidate the potential interactions of gemcitabine and taxanes in combination chemotherapy regimens.

It is important to acknowledge and investigate the role of the characteristic dense, stroma-rich microenvironment in which PDAC cells grow. Cancer-associated fibroblasts (CAFs), cells that define the PDAC stroma, have been shown to play a role in decreasing patient sensitivity to chemotherapeutic treatment in breast, lung, esophageal, and colorectal cancer (20-22). While PDAC cell lines overall exhibited a more dramatic reduction in chemosensitivity to Gem-Pac than to FOLFIRINOX when cultured with fibroblast-conditioned media, different cell lines exhibited a heterogeneous response to therapy, with several lines remaining unaffected by the presence of fibroblastconditioned media. The diversity of CAF subtypes may, therefore, play a role in eliciting this heterogeneous response.

Understanding that chemotherapy impacts the growth and proliferation of both tumor and stromal cells, we also evaluated the impact of PDAC cell line conditioned media on fibroblast response to therapy. When treated in PDAC cell line conditioned media, all fibroblast lines exhibited a decrease in sensitivity to both drug regimens. Developing a better understanding of how the PDAC tumor microenvironment impacts the efficacy of different chemotherapeutics could help inform the nuances of PDAC tumor biology, as well as tailor treatment decisions for patients. 
A

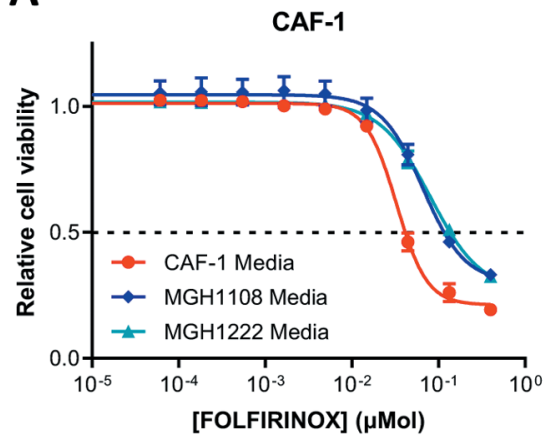

D

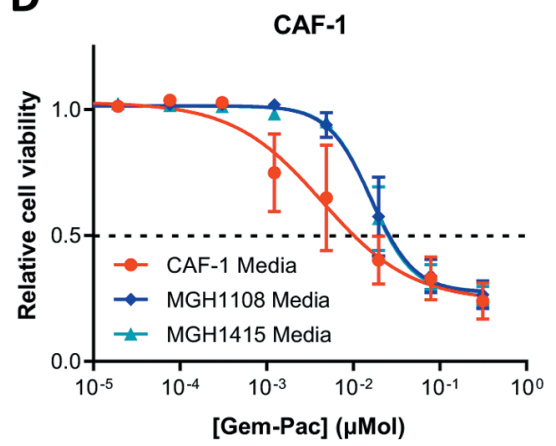

B

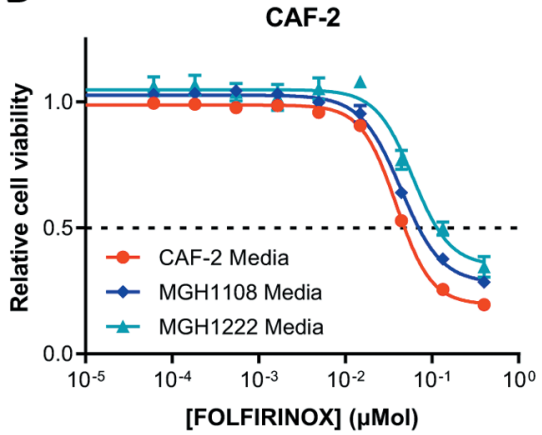

E

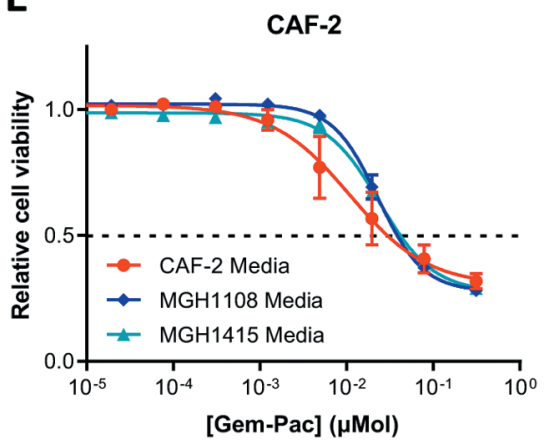

C

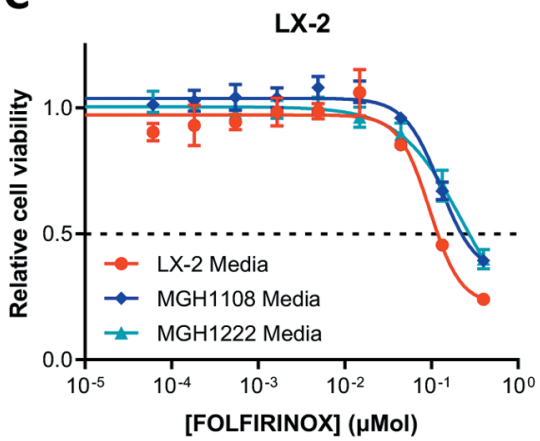

$\mathbf{F}$

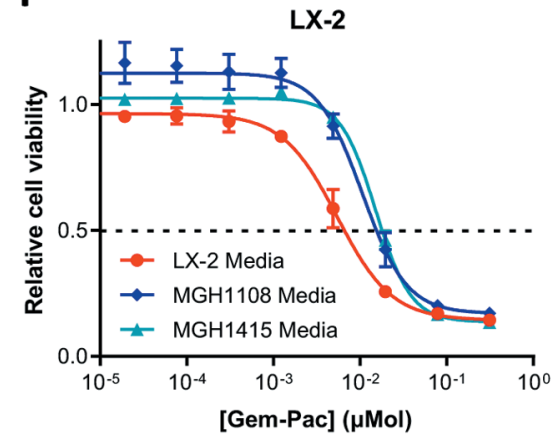

Figure 5. Effect of PDAC-conditioned media on fibroblast chemosensitivity. (A) Dose-response curves for PDAC-derived CAF-1 treated with FOLFIRINOX in the presence of conditioned media from CAF-1 and PDAC-derived cell lines MGH1108 and MGH1222. (B) Dose-response curves for PDAC-derived CAF-2 treated with FOLFIRINOX in the presence of conditioned media from CAF-2, MGH1108, and MGH1222. (C) Dose-response curves for hepatic derived LX-2 treated with FOLFIRINOX in the presence of conditioned media from LX-2, MGH1108, and MGH1222. (D) Dose-response curves for CAF-1 treated with gemcitabine plus paclitaxel (Gem-Pac) in the presence of conditioned media from CAF-1, MGH1108, and PDAC-derived cell line MGH1415. (E) Dose-response curves for CAF-2 treated with Gem-Pac in the presence of conditioned media from CAF-2, MGH1108, and MGH1415. (F) Dose-response curves for LX-2 treated with Gem-Pac in the presence of conditioned media from LX-2, MGH1108, and MGH1415.

With improved understanding of the intrinsic heterogeneity of pancreatic cancer in different patients, these findings support the need for future research towards customizing treatments to fit the diverse needs of these individuals. Cell lines may represent a unique tool for stratifying patient chemosensitivity; further proteomic and transcriptomic analyses of the cell lines used in our study could help identify biomarkers that ultimately guide the use of one drug regimen over the other for a given patient. These lessons learned from patient-derived PDAC cell lines could additionally help evaluate the validity of other recently published biomarkers that purport to predict chemotherapeutic response.

\section{Conflicts of Interest}

The Authors have no conflicts of interest to declare.

\section{Authors' Contributions}

ASL and CFC conceived the study. SKSB, DJB, CFC, MMM, and ASL participated in the design of the study. SKSB and DJB performed the experiments. SKSB, DJB, JWC, KDL, ALW, CFC, and ASL analyzed and/or interpreted the data. UFW and OS generated CAF-2 cells used in this study. SKSB and DJB wrote the manuscript under the supervision of ASL with input from all authors.

\section{Acknowledgements}

While at MGH, DJB was supported by a fellowship from the ARC Foundation (https://www.fondation-arc.org/).

\section{References}

1 Conroy T, Desseigne F, Ychou M, Bouché O, Guimbaud R, Bécouarn Y, Adenis A, Raol J-L, Gourgou-Bourgade S, de la Fouchardière C, Bennouna J, Bachet JB, Khemissa-Akouz F, PéréVergé D, Delbaldo C, Assenat E, Chauffert B, Michel P, MontotoGrillot $\mathrm{C}$ and Ducreux M: FOLFIRINOX versus gemcitabine for metastatic pancreatic cancer. N Engl J Med 364(19): 1817-1825, 2011. PMID: 21561347. DOI: 10.1056/NEJMoa1011923

2 Von Hoff DD, Ervin T, Arena FP, Chiorean EG, Infante J, Moore M, Seay T, Tjulandin SA, Ma WW, Saleh MN, Harris M, Reni M, Dowden S, Laheru D, Bahary N, Ramanathan RK, Tabernero J, Hidalgo M, Goldstein D, Van Cutsem E, Wei X, Iglesias J and 
Renschler MF: Increased survival in pancreatic cancer with nabpaclitaxel plus gemcitabine. N Engl J Med 369(18): 1691-1703, 2013. PMID: 24131140. DOI: 10.1056/NEJMoa1304369

3 Quinn BA, Lee NA, Kegelman TP, Bhoopathi P, Emdad L, Das SK, Pellecchia M, Sarkar D and Fisher PB: The quest for an effective treatment for an intractable cancer: Established and novel therapies for pancreatic adenocarcinoma. Adv Cancer Res 127: 283-306, 2015. PMID: 26093904. DOI: 10.1016/bs.acr.2015.04.009

4 Plunkett W, Huang P, Xu YZ, Heinemann V, Grunewald R and Gandhi V: Gemcitabine: metabolism, mechanisms of action, and self-potentiation. Semin Oncol 22(4 Suppl 11): 3-10, 1995. PMID: 7481842 .

5 Weaver BA: How Taxol/paclitaxel kills cancer cells. Mol Biol Cell 25(18): 2677-2681, 2014. PMID: 25213191. DOI: 10.1091/mbc.E14-04-0916

6 Narayanan V and Weekes C: Nanoparticle albumin-bound (nab)paclitaxel for the treatment of pancreas ductal adenocarcinoma. Gastrointest Cancer Targets Ther 5: 11-19, 2015. DOI: 10.2147/GICTT.S55158

7 Heinrich $\mathrm{S}$ and Lang $\mathrm{H}$ : Neoadjuvant therapy of pancreatic cancer: definitions and benefits. Int J Mol Sci 18(8): E1622, 2017. PMID: 28933761. DOI: 10.3390/ijms18081622s

8 Ducreux M, Cuhna AS, Caramella C, Hollebecque A, Burtin P, Goéré D, Seufferlein T, Haustermans K, Van Laethem JL, Conroy $\mathrm{T}$ and Arnold D: Cancer of the pancreas: ESMO Clinical Practice Guidelines for diagnosis, treatment and follow-up. Ann Oncol 26(Suppl 5): v56-68, 2015. PMID: 26314780. DOI: 10.1093/annonc/mdv295

9 Kamisawa T, Wood LD, Itoi T and Takaori K: Pancreatic cancer. Lancet 388(10039): 73-85, 2016. PMID: 26830752. DOI: 10.1016/S0140-6736(16)00141-0

10 Khorana AA, Mangu PB, Berlin J, Engebretson A, Hong TS, Maitra A, Mohile SG, Mumber M, Schulick R, Shapiro M, Urba S, Zeh HJ and Katz MHG: Potentially curable pancreatic cancer: American society of clinical oncology clinical practice guideline update. J Clin Oncol 35(20): 2324-2328, 2017. PMID: 28398845. DOI: $10.1200 /$ JCO.2017.72.4948

11 Ryan DP, Hong TS and Bardeesy N: Pancreatic adenocarcinoma. N Engl J Med 371(11): 1039-1049, 2014. PMID: 25207767. DOI: $10.1056 /$ NEJMra1404198

12 Sohal DPS, Mangu PB, Khorana AA, Shah MA, Philip PA, O'Reilly EM, Uronis HE, Ramanathan RK, Crane $\mathrm{CH}$, Engebretson A, Ruggiero JT, Copur MS, Lau M, Urba S and Laheru D: Metastatic pancreatic cancer: American Society of Clinical Oncology clinical practice guideline. J Clin Oncol 34(23): 2784-2796, 2016. PMID: 27247222. DOI: 10.1200/JCO.2016.67.1412

13 Conroy T, Hammel P, Hebbar M, Ben Abdelghani M, Wei AC, Raoul, J-L, Choné L, Francois E, Artru P, Biagi JJ, Lecomte T, Assenat E, Faroux R, Ychou M, Volet J, Sauvanet A, Breysacher G, Di Fiore F, Cripps C, Kavan P, Texereau P, Bouhier-Leporrier K, Khemissa-Akouz F, Legoux JL, Juzyna B, Gourgou S, O'Callaghan CJ, Jouffroy-Zeller C, Rat P, Malka D, Castan F and Bachet JB: FOLFIRINOX or gemcitabine as adjuvant therapy for pancreatic cancer. N Engl J Med 379(25): 2395-2406, 2018. PMID: 30575490. DOI: 10.1056/NEJ Moa1809775
14 Zhang C, Xu Y, Wang H, Li G, Yan H, Fei Z, Xu Y and Li W: Curcumin reverses irinotecan resistance in colon cancer cell by regulation of epithelial-mesenchymal transition. Anticancer Drugs 29(4): 334-340, 2018. PMID: 29420338. DOI: 10.1097/CAD.0000000000000599

15 Menéndez JA, Del Mar Barbacid M, Montero S, Sevilla E, Escrich E, Solanas M, Cortés-Funes H and Colomer R: Effects of gamma-linolenic acid and oleic acid on paclitaxel cytotoxicity in human breast cancer cells. Eur J Cancer 37(3): 402-413, 2001. PMID: 11239764. DOI: 10.1016/s0959-8049(00)00408-1

$16 \mathrm{Ma}$ WW and Hidalgo M: The winning formulation: The development of paclitaxel in pancreatic cancer. Clin Cancer Res 19(20): 5572-5579, 2013. PMID: 23918602. DOI: 10.1158/ 1078-0432.CCR-13-1356

17 Maitra A and Hruban RH: Pancreatic cancer. Annu Rev Pathol Mech Dis 3: 157-188, 2008. PMID: 18039136. DOI: 10.1146/ annurev.pathmechdis.3.121806.154305

18 Chan KKW, Guo H, Cheng S, Beca JM, Redmond-Misner R, Isaranuwatchai W, Qiao L, Earle C, Berry SR, Biagi JJ, Welch S, Meyers BM, Mittmann N, Coburn N, Arias J, Schwartz D, Dai WF, Gavura S, McLeod R and Kennedy ED: Real-world outcomes of FOLFIRINOX vs gemcitabine and nab-paclitaxel in advanced pancreatic cancer: A population-based propensity score-weighted analysis. Cancer Med 9(1): 160-169, 2020. PMID: 31724340. DOI: 10.1002/cam4.2705

19 Lacobuzio-Donahue CA, Velculescu VE, Wolfgang CL and Hruban RH: The genetic basis of pancreas cancer development and progression: insights from whole-exome and whole-genome sequencing. Clin Cancer Res 18(16): 4257-4265, 2012. PMID: 22896692. DOI: 10.1158/1078-0432.CCR-12-0315

20 Liu ML, Zang $\mathrm{F}$ and Zhang SJ: RBCK1 contributes to chemoresistance and stemness in colorectal cancer (CRC). Biomed Pharmacother 118: 109250, 2019. PMID: 31545242. DOI: $10.1016 /$ j.biopha.2019.109250

21 Su S, Chen J, Yao H, Liu J, Yu S, Lao L, Wang M, Luo M, Xing Y, Chen F, Huang D, Zhao J, Yang L, Liao D, Su F, Li M, Liu Q and Song E: CD10+GPR77+ cancer-associated fibroblasts promote cancer formation and chemoresistance by sustaining cancer stemness. Cell 172(4): 841-856.e16, 2018. PMID: 29395328. DOI: 10.1016/j.cell.2018.01.009

22 Tanaka K, Miyata H, Sugimura K, Fukuda S, Kanemura T, Yamashita K, Miyazaki Y, Takahashi T, Kurokawa Y, Yamasaki M, Miyazaki Y, Takahashi T, Kurokawa Y, Yamasaki M, Wada H, Nakajima K, Takiguchi S, Mori M and Doki Y: miR-27 is associated with chemoresistance in esophageal cancer through transformation of normal fibroblasts to cancer-associated fibroblasts. Carcinogenesis 36(8): 894-903, 2015. PMID: 26026166. DOI: $10.1093 /$ carcin/bgv067
Received April 8, 2020

Revised May 18, 2020

Accepted May 20, 2020 